A R T I C ULO

\title{
REFLEXIONES SOBRE LA FAMILIA
}

\section{A PARTIR DE LA EXPLicación HistóRICA \\ DE la Organización Social ACtual}

\author{
Por: Francisco Chacón Jiménez
}

Artículo Recibido: Abril 16 de 2008

Artículo Aceptado: Junio 18 de 2008

\section{RESUMEN}

El artículo presenta reflexiones acerca de la familia y su devenir histórico, tras treinta años de estudio del hogar. Para ello señala algunos aspectos teórico-metodológicos a partir de la incorporación del ciclo de vida, el modo de acceso y distribución de los recursos materiales y la relación tipología del hogar-sistemas de herencia, hasta llegar a plantear las principales contradicciones y revoluciones que hoy en día se suscitan a su interior.

Palabras Claves: Familia, ciclo vital, evoluciones generacionales y cambios genealógicos.

\begin{abstract}
The article reflexionates about family and it's historic trajectory after thirty years of research in the subject. It shows some theorical and methodological aspects relative to the incorporation of concepts like life cycle, acces and distribution of material resources and the relationship between the tipology of the home and the succession systems, also explaining the main contradictions and revolutions developed inside the families.
\end{abstract}

Key Words: Family, vital cycle, generational evolutions and genealogical changes.

\section{Introducción}

Articulo tipo 2, de Reflexión, según clasificación Colciencias.
Yada sociedad y cada época plantean sus prioridades. Y las del espacio iberoamericano se encuentran muy condicionadas por la fuerte y potente tradición de la familia y su notable repercusión en la explicación de la organización social contemporánea así como en sus contradicciones, conflictos, 
proyección y perspectivas vitales de las personas que integran dichas poblaciones. Además, las sociedades contemporáneas se interrogan, incluso con notable preocupación e inquietud, por su futuro. La complejidad y las contradicciones de los sistemas sociales son los responsables de esta especie de "angustia" vital de origen y significación social respecto a la comprensión del mundo en el que se vive.

Y cuando el ciudadano/a mira a su alrededor observa, por una parte derechos y legislación, asistencia y protección pública, pero los efectos, los sentimientos, la confianza, el amor, el cariño, el sentimiento de ser individuo y persona en tanto que tiene referencias sólo se encuentra en la familia y a partir de su familia; si esta situación no existe la exclusión y la marginación asoman por la puerta social.

Los historiadores latinoamericanos y quienes trabajan sobre este espacio regional deben asumir la responsabilidad de mirar a las respectivas sociedades con ojos atentos en la medida en que han asimilado el mandato social y encontrado condiciones idóneas para desarrollar su capacidad de reflexión. Como indica el historiador cubano Oscar Zanetti y recoge Ana Vera, permanecen zonas del conocimiento insuficientemente exploradas y continúan repitiéndose ideas adocenadas y superadas sobre tópicos que demandan mayor profundidad y prolijidad de investigaciones, aunque las agendas de los historiadores de cada país y sobre cada país no pueden ser iguales totalmente a otros países y espacios regionales, sean americanos o europeos. En este contexto resulta curioso que en la obra Los métodos de la historia de Ciro F.S. Cardoso y Héctor Pérez Brignoli, publicada por primera vez en 1976, con tan gran repercusión en América Latina, y excelente para señalar el estado de la metodología y de la historiografía, se aprecia una ausencia total de la historia de la familia. La fuerte influencia hegemónica en el ámbito internacional de Annales y del materialismo histórico, ocultó el papel trascendental del individuo y a la vez de la colectividad y, sobre todo, de los lazos, vínculos y redes de relación social, familiar y profesional para explicar tanto la reproducción del sistema social como de los cambios y continuidades.

La familia constituye no solo una de las preocupaciones sociales y políticas de los gobiernos actuales, sino el primer punto de referencia e interés para los propios ciudadanos. Sin embargo, el proceso evolutivo seguido en España y América Latina respecto al papel de la mujer, el matrimonio, las nuevas formas de familia, la nuclearización de los hogares, el aumento del número de ancianos en relación con la fuerte disminución de las tasas de natalidad o las corrientes 
migratorias y las desigualdades sociales, no sólo registra un enorme desequilibrio sino que son factores complementarios y estrechamente relacionados.

Dichas tareas se vuelven perentorias y totalmente urgentes si queremos atender las siguientes necesidades: 1) explicar determinadas problemáticas, como la herencia, la mayor o menor fecundidad, el proceso de envejecimiento, o las transformaciones en el parentesco analizando la evolución que han seguido y comparando dicha situación con la existente en América Latina. 2) Estudiar el relevante papel que ha tenido la mujer en estas transformaciones. Y todo ello dentro de tres vertientes: a) la incorporación del ciclo de vida, b) el modo de acceso y distribución de los recursos materiales, c) la relación tipología del hogar-sistema de herencia.

La perspectiva comparativa, constituye en la historiografía internacional actual uno de los enfoques más positivos en la creación de conocimiento. Determinar las influencias de determinados aspectos y los elementos comunes a las sociedades de los distintos países, es un factor fundamental en el análisis de los procesos sociales, especialmente si se refiere al ámbito de las ciencias sociales y, en concreto, al de la familia y la cultura.

\section{Aspectos Teóricos y Metodológicos}

La incorporación del ciclo de vida, con lo que supone de descubrimiento del individuo. En este punto, las fuentes obligan a una revisión. Especialmente en el caso de los censos, instrumento básico para el Grupo de Cambridge. De éstos, más allá de sus posibilidades y de los códigos que sus datos contienen, se ha pretendido obtener informaciones, por ejemplo, sobre las relaciones de parentesco y de trabajo que sólo a partir de lo nominativo y del cruce y complementariedad con fuentes parroquiales, notariales o judiciales es posible. La relevancia, nueva lectura, cruce e incorporación de nuevas fuentes materiales e inmateriales, es una llamada de atención ante las perspectivas integradoras que el objeto de investigación plantea en el análisis explicativo de la organización social. Si la mirada rígida y estática a las fuentes originó conclusiones, hasta cierto punto, distorsionantes de la realidad social, es necesario corregir este tipo de enfoques mediante un cruce de fuentes que tenga un punto de confluencia y de reunión de esa diversidad. Y ese lugar y espacio de reunión no es otro que: el nombre.

Lo nominativo $\mathrm{y}$, por tanto, la recuperación del individuo en trayectorias personales dentro de grupos familiares integrados en genealogías, ofrece 
una dimensión analítica necesaria y hasta se diría que obligada. No se trata de realizar una reconstrucción de familias siguiendo la técnica de L. Henry, sino una reconstrucción genealógica, como están haciendo los investigadores portugueses.

El modo de acceso y distribución de los recursos materiales entre los distintos miembros de las unidades familiares y su influencia sobre las formas de residencia. Aquí se ponen en juego los sistemas de transmisión (herencia, dote, donación, compra-venta), el orden de nacimiento y, sobre todo, las prácticas de acceder al matrimonio antes o después de la muerte de los padres y en qué momento: matrimonio hijos/as, o bien muerte de los padres, y en qué cantidad o proporción (todo o una parte), se transmite. Sin embargo, el concepto recurso debe entenderse como algo más que paso y cesión a la siguiente generación de la herencia familiar. Debemos entenderlo como un sistema de relaciones de producción a partir del cuál se ponen en marcha las distintas estrategias de reproducción social. Y ha significado que muchos autores, al estudiar los sistemas económicos y políticos hayan tenido que hacer referencia a la familia para explicar el funcionamiento de los mismos.

Un resultado señalado por diferentes especialistas es incorporar los comportamientos diferenciados de los distintos sectores sociales. Lo que nos conduce a los problemas de dominación, desigualdad, dependencia y jerarquización, tan característicos de las sociedades tradicionales y que se encontraban plenamente imbricados e integrados en las relaciones sociales de producción cuyos actores y protagonistas son la familia y componen lo esencial del tejido social.

Pero si se ha avanzado en el conocimiento de la realidad social a través de la familia, es por la aplicación del concepto: capital relacional. Se trata de un sistema de relaciones que permite la integración y comparabilidad entre factores distintos. Así, la capacidad y posibilidades de un sistema relacional que compara e integra a la vez aspectos estudiados, hasta ahora, sectorialmente, es una de las nuevas propuestas.

La relación tipología del hogar-sistemas de herencia explicaría tres situaciones con las que es necesario contar a la hora de explicar el significado y la influencia de la familia en la organización social y política de una comunidad.

- Adaptabilidad ante situaciones de crecimiento o de crisis. El ejemplo más claro es, precisamente, la familia troncal y su 
supervivencia frente al código civil.

- La casa se mueve. Es decir, la necesidad de integración de las nuevas unidades familiares en una de las familias de origen por necesidad, obliga a reformas físicas de la casa y a una convivencia que significa compartir espacios comunes básicos, como es la cocina o la mesa donde tradicionalmente se realiza la comida principal de la familia. El ciclo de vida y la coyuntura demográfica que le acompaña va unido a este proceso de adaptabilidad espacial y social.

- La neolocalidad. Estamos ante una práctica en el momento de la constitución de nuevos hogares, que no determina por sí misma la existencia de una familia nuclear en el sentido pleno de independencia y autonomía de la nueva unidad doméstica. En muchas ocasiones las relaciones de parentesco y de trabajo explican una relación más allá de la división que el censo como fuente indica y que la realidad social oculta en aquel. Nuevamente el ciclo de vida y la trayectoria familiar con la coyuntura demográfica explica situaciones que nunca pueden ser entendidas de manera estática.

Este podría ser el resultado de la relación entre los sistemas de herencia y las formas familiares a partir del estudio de las tipologías del hogar; uno de los enfoques básicos en el estudio de la Familia a lo largo de su proceso histórico y actual.

En definitiva, trabajo, parentesco, continuidad generacional y ciclo de vida matizan el sistema de residencia en el contexto de unas solidaridades que se reflejan en lazos, vínculos de una fuerza y resistencia muy notables y, por tanto, básicos para comprender la sociedad y su proceso histórico a través de la familia.

\section{Contradicciones y Revoluciones}

La familia atraviesa coyunturas históricas que le influyen a la vez que la realidad familiar soporta dichas coyunturas. Tres grandes procesos civilizatorios debemos de considerar: a) el paso de lo privado a lo público, b) de lo religioso a lo profano y c) de la familia al Estado. Las interinfluencias en estos procesos tienen siempre a la familia como el marco en el que se producen, ya que se trata de derechos civiles que refrendados por la legislación correspondiente permite actuar en el seno de lo individual y de lo familiar. 
Un ejemplo de estas constantes y fundamentales interinfluencias es la siguiente contradicción: la familia ha ido abandonando responsabilidades en tanto que el Estado ha ido asumiéndolas; de tipo escolar y educativo, profesional y, por supuesto, en la regulación hereditaria a través de la promulgación de códigos civiles. Lo cuál no significa que la familia haya perdido su capacidad de núcleo aglutinante en la constitución y funcionamiento de la organización social. Ahora bien, es evidente que, a partir de los derechos civiles que los individuos han ido adquiriendo como reconocimiento de su personalidad e individualidad en el conjunto de la sociedad, se han producido una serie de adaptaciones y de cambio de responsabilidades en el seno de la institución familiar, pero que no han disminuido el papel básico de la célula de funcionamiento de la sociedad. Sería un error oponer Familia a Estado, pero sí subrayar la paulatina transferencia de responsabilidades a la vez que se ganan derechos individuales de carácter civil.

Una segunda contradicción se puede apuntar cuando se analiza la actuación de las políticas públicas que tratan de influir sobre los ciudadanos para conseguir una mejor situación de las familias; en estos casos, algunas de las medidas que se acuerdan no dependen de la capacidad de los individuos, o bien se tienen que poner en práctica en el plano privado; sin embargo generan y crean las condiciones necesarias para poder desarrollar y conseguir los objetivos propuestos. En definitiva, se trata de la dinámica privado-público, que como proceso civilizatorio encierra una latente contradicción. Pese a estas contradicciones la familia no desaparece, se adecúa, se transforma y los ciudadanos siguen integrados en la célula social pero con otras posibilidades.

A estas dos contradicciones le corresponden dos revoluciones: a) no es necesario casarse para formar una familia, ni tampoco la unión de dos personas de sexo distinto para la reproducción biológica y la construcción de la unidad familiar. B) La mujer puede decir no. Es decir, ha conseguido la autonomía personal, y la no dependencia del padre o del marido; naturalmente por medio de la formación, el acceso a los puestos de trabajo y la capacidad e independencia económica.

Pero estas revoluciones sólo son posibles y se producen si la sociedad lo permite y las acepta, y formarán parte indisociable de la organización social. La enseñanza histórica y actual de la familia es que ésta no puede seguir una evolución independiente y sin relación con los valores culturales y la idiosincrasia de esa sociedad. Por ello las comparaciones deben hacerse con mucha prudencia.

Hoy, tanto la mujer como el hombre quieren tener su propio proyecto profesional 
y vital y, por tanto, independiente. Sin embargo, las poblaciones dependientes aumentan como consecuencia de la constante caída de la fecundidad, lo que produce una más elevada tasa de envejecimiento. Las situaciones son muy distintas según los grupos sociales; las desigualdades suelen ser frecuentes y pronunciadas cuando no existe Estado que apoye a la familia, o bien aquel no se preocupa de determinados aspectos porque, por natural, la familia lo hace. Los valores culturales de apoyo y cuidado de la familia no forman parte actualmente por parte del joven o la joven, al menos como responsabilidad interiorizada y asumida. Aquí se produce una ruptura con la tradición. Cuando estas variables se agudizan, la situación se agudiza más y surgen problemas que deben ser identificados: a) problemas de mercado laboral para la mujer; b) acceso a la vivienda para jóvenes; c) dificultad de conciliar la vida familiar y laboral; d) situaciones de violencia de género en el seno de la familia y de las relaciones personales.

La familia, como célula básica de organización social, no es ajena a estas profundas transformaciones. Al contrario, está en la punta de lanza de estos cambios, es un espejo donde se refleja la evolución de un país.

Resulta evidente la interacción entre la institución familiar y la realidad política, económica y social. Las tendencias hacia una mayor democratización de las relaciones sociales, la creciente incorporación de las mujeres al mercado de trabajo, los cambios y la diversificación en los modelos de organización familiar, la evolución de las variables sociodemográficas, las mayores demandas de flexibilidad por parte de una economía cada vez más globalizada, son, en palabras de Félix Barajas a quien seguimos en estos razonamientos que a pesar de ser referidos al caso de España son válidos para otros espacios, elementos que configuran un nuevo panorama para las familias, en abierta contradicción con el modelo tradicional de organización familiar basado en el reparto de funciones por género.

Los cambios sociales, políticos, demográficos, culturales y económicos de gran magnitud a que se ha venido refiriendo han afectado de manera directa a la familia. La democracia y la modernidad, han revolucionado la institución familiar desde fuera y por dentro. Se ha dado el paso de la unidad a la diversidad. Los sistemas autoritarios se caracterizan por promover modelos unívocos y estáticos, donde cada cosa y cada quien tiene su sitio predeterminado. La libertad, por el contrario, conlleva complejidad y pluralismo y los ciudadanos deciden de forma cada vez más libre de presiones sociales cómo organizan su convivencia y cómo viven su dimensión familiar. El modelo tradicional de familia basado en el reparto de roles por género y en la jerarquía patriarcal ha quebrado social y legalmente. El 
nuevo paradigma familiar se basa en los principios democráticos de libertad e igualdad entre sus miembros. Pero estos procesos no se han producido sin costes ni resistencias.

\section{Conclusión}

La familia era y es una manera de pensar, una práctica propiciada por ciertos valores, es decir, un habitus más que una estructura formal o una institución. Naturalmente, tiene una connotación y realidad institucional y adopta unas formas que son cambiantes en función de las coyunturas históricas que se reflejan en los nacimientos, defunciones y matrimonios. También la familia es una representación social expresada mediante una descripción que recoge la morfología y las funciones. El modelo familiar es una articulación entre el orden biológico y el orden social, y hay relación directa entre el modo de concebir la familia y los comportamientos. La etnología y la antropología han enseñado a considerar la familia como una construcción cultural y como la unidad elemental de la función social. La familia es, pues, un sistema de relación con el contexto social y red de relaciones comunitarias.

Es cierto que la familia, desde el punto de vista de la persona individual es algo transitorio, pero la tumba y la capilla ofrecen, aparte de una entidad física y precisa, la permanencia y la perpetuidad que la familia necesita. Los elementos simbólicos alcanzan un grado de concreción que pasan a ser conocidos por el resto de la población. Pero existen cuatro peligros: a) considerar la familia estadísticamente y en términos estáticos y de aislamiento; b) confundir familia con hogar o agregado doméstico; c) no considerar en el análisis de la familia su plasticidad, ni los cambios o transformaciones sociales que se producen a partir del grupo de parentesco; d) ignorar género y clase.

La historia de la familia se vinculó excesivamente a la demografía histórica. Sin embargo, el marco teórico y metodológico se ha ido ampliando hasta colocar los objetivos en el horizonte de la organización social y su proceso explicativo. Ello ha obligado a una revisión de las fuentes y a tener presente tres principios: 1) el contexto teórico y metodológico, ya que la investigación de los últimos treinta años abandonó el contexto y no tuvo en cuenta la familia sino el hogar; 2) repensar las fuentes en perspectiva nominativa $y$, en consecuencia, genealógica y 3) conocimiento y explicación de la organización social.

La familia valora más, actualmente, las relaciones personales que conducen a 
realizaciones profesionales y a la búsqueda de la felicidad que la conservación, transmisión de bienes y otorgar nombre o perpetuar memoria y antepasados. Se insiste e invierte mucho más en la vida privada, cuando es lo público lo que permite ser atendidos. Ha perdido fuerza el sentido de institución. En este contexto, el matrimonio era la supervivencia del grupo y todo individuo se integraba en esta lógica. La institución del matrimonio y de la familia aparecía como un hecho natural. Era una práctica cotidiana, plenamente interiorizada; lo cuál no quiere decir que no surgiese el conflicto, el maltrato e intentos de separación y divorcios, pero no una alternativa hacia otras formas familiares.

Pero la conclusión más notable a la que se puede llegar es la de que asistimos, tras treinta años de estudio hegemónico del hogar, a la superación del mismo y al protagonismo del conjunto $y$, por tanto, de la familia, pero dentro de la importancia y trascendencia del individuo. Es por ello que los protagonistas y sus conductas se individualizan, pero en el interior de familias que son analizadas en términos de ciclo de vida, evoluciones generacionales y cambios genealógicos.

\section{BIBLIOGRAFÍA}

BALMORI, D., VOSS, S.F., y WORTMAN, M. (1990). Las alianzas de familias y la formación del país en América Latina. Fondo de Cultura Económica de México. México.

BARAJAS VILLALUENGA, F. (2008). Texto inédito intervención Máster "Historia comparada: pasado y presente de las relaciones sociales, familiares y de género en Europa y América Latina", 12 septiembre-2008.

CASEY, J. (1990). Historia de la Familia. Espasa Calpe. Madrid.

CHACÓN JIMÉNEZ, F., et. al. (2003). Sin distancias. Familia y tendencias historiográficas en el siglo XX. col. Mestizo. Universidad de Murcia-Universidad Externado de Colombia. Murcia.

CHACÓN JMÉNEZ, F. y HERNÁNDEZ FRANCO, J. (2007). Espacios sociales, universos familiares. La familia en la historiografía española. Editum. Universidad de Murcia. Murcia.

CHACÓN JMÉNEZ, F. (2008). La revisión de la tradición: prácticas y discurso en la nueva historia social. En: Historia Social, 60, págs. 145-154. 
CHACÓN JIMÉNEZ, F. (en prensa). Familia y hogar en la sociedad española. Mitos y realidades históricas.

DELILE, G. (2003). Le maire et le prieur. Pouvoir central et pouvoir local en Mediterranée occidentale (XVé-XVIIIé siécle). Ecole des Hautes Etudes en Sciences Sociales. Paris.

DIARIO OFICIAL DE LA UINIÓN EUROPEA, 14 MARZO 2007. Dictamen del Comité Económico y Social Europeo sobre la familia y la evolución demográfica.

INSTITUTO DE POLÍTICA FAMILIAR. (2006). Informe Evolución de la Familia en Europa.

MILANICH, N. (2007). "Whither family history? A Road map from Latin America", Review Essay, vol. 113, 2 april, págs. 439-457.

MOLINIÉ BERTRAND, A. y RODRÍGUEZ JIMÉNEZ, P. (eds.). (2000). A través del tiempo. Diccionario de fuentes para la historia de la familia, col. Mestizo. Universidad de Murcia. Murcia.

RODRÍGUIEZ JMÉNEZ, P. (Coord.). (2004). La familia en Iberoamérica 1550-1980. Universidad Externado de Colombia. Bogotá.

VERA ESTRADA, A. y ROBICHAUX, D. (Comp.). (2008). Familias y culturas en el espacio Latinoamericano. Universidad Iberoamericana-Centro de Investigación y Desarrollo de la cultura cubana Juan Marinello. México.

\section{BIOGRAFIA}

\section{FRANCISCO CHACÓN JIMÉNEZ}

Licenciado y Doctor en Historia (Universidad de Murcia). Profesor catedrático de Historia Moderna de la Universidad de Murcia (España). Sus líneas de investigación son Historia Local y Regional e Historia Social y de la Familia. Dirige la Fundación Centro de Estudios Históricos e Investigaciones Locales Región de Murcia. Vicepresidente de las Asociaciones Profesionales de Historia Social y de Historia Moderna. Entre sus libros más recientes están: Poder, Familia y Consanguinidad en la España del Antiguo Régimen, (1992), Familia, Poderosos y Oligarquías, (2000) y Sin distancias. Familia y tendencias historiográficas en el siglo XX. (2002). Integrante Grupo de Investigación Estudios de Familias, Masculinidades y Feminidades. Universidad de Cartagena (Colombia).

e-mail: chaconmu@um.es 